This item was submitted to Loughborough's Research Repository by the author.

Items in Figshare are protected by copyright, with all rights reserved, unless otherwise indicated.

The essential interactions in oxides and spectral weight transfer in doped manganites

PLEASE CITE THE PUBLISHED VERSION

LICENCE

CC BY-NC-ND 4.0

REPOSITORY RECORD

Alexandrov, A.S., and A.M. Bratkovsky. 2019. "The Essential Interactions in Oxides and Spectral Weight Transfer in Doped Manganites”. figshare. https://hdl.handle.net/2134/1205. 


\title{
The essential interactions in oxides and spectral weight transfer in doped manganites
}

\author{
A.S. Alexandrov ${ }^{1, *}$ and A.M. Bratkovsky ${ }^{2, \dagger}$ \\ ${ }^{1}$ Department of Physics, Loughborough University, Loughborough LE11 3TU, UK \\ ${ }^{2}$ Hewlett-Packard Laboratories, 3500 Deer Creek Road, Palo Alto, California 94304-1392
}

(August 11, 1999)

\begin{abstract}
We calculate the value of the Fröhlich electron-phonon interaction in manganites, cuprates, and some other charge-transfer insulators and show that this interaction is much stronger than any relevant magnetic interaction. A polaron shift due to the Fröhlich interaction, which is about $1 \mathrm{eV}$, suggests that carriers in those systems are small (bi)polarons at all temperatures and doping levels, in agreement with the oxygen isotope effect and other data. An opposite conclusion, recently suggested in the literature, is shown to be incorrect. The frequency and temperature dependence of the optical conductivity of ferromagnetic manganites is explained within the framework of the bipolaron theory.
\end{abstract}

71.38.+i, 74.20.Mn, 75.30.Vn, 78.20.-e

\section{INTRODUCTION}

It is well understood that carriers in cuprates 11.2 and manganites [3 5] are strongly coupled to lattice vibrations. We have recently proposed a bipolaron theory of ferromagnetism and colossal magnetoresistance (CMR) based on the idea of a current carrier density collapse (CCDC) due to an interplay of the electron-phonon and exchange interactions in doped manganites [5]. Owing to the strong electron-phonon interaction, polaronic carriers are bound into almost immobile bipolarons in the paramagnetic phase of CMR materials. The non-degenerate polarons induce a polarization of localized $\mathrm{Mn} d$ electrons. As a result, the exchange interaction dissociates bipolarons below $T_{c}$ if the $p-d$ exchange energy $J_{p d} S$ of the polaronic carriers with the localized Mn $d$ electrons is larger than the bipolaron binding energy $\Delta$. Hence, the density of current carriers (polarons) suddenly increases below $T_{c}$, which explains the resistivity peak and CMR, observed in many ferromagnetic oxides 6 , 8. We have shown [9] that CCDC also explains the giant isotope effect [10,11], the tunneling gap [12], the specific heat anomaly 13, and the temperature dependence of the dc resistivity 14.

The non-metallic nature of the ferromagnetic lowtemperature phase of the doped manganites has been unambiguously confirmed in recent studies of the optical conductivity [15 18] and photoemission [19. In particular, a broad incoherent spectral feature [15 18 in the midinfrared region and a pseudogap in the excitation spectrum 19 were observed, while the coherent Drude weight appeared to be one or even two orders of magnitude smaller 16, 17 than expected for a metal, or almost absent [18]. These and other studies [20] prove that carriers retain their polaronic character well below $T_{c}$, in agreement with our theory of CMR [5]. For example, the measured residual conductivity (at $T=0$ ) $\sigma_{0}=360 \Omega^{-1} \mathrm{~cm}^{-1}$ [18] yields the product of the Fermi wave vector and the mean free path $k_{F} l \lesssim 1$, which is below the Ioffe-Regel limit [21]. Hence, Fermi-liquid type theories appear to be inadequate even for a description of the low-temperature phase of manganites.

Among the major phenomena yet to be explained in the manganites are the spectral weight transfer with temperature 16] 18] and the pronounced peak structure [18] in the optical conductivity both above and below $T_{c}$, observed in several systems.

In this paper we first calculate the value of the electronphonon interaction in oxides. We then propose a theory of the optical conductivity, including the massive spectral weight transfer below the ferromagnetic transition, based on the idea of the current carrier density collapse. We show that the high-temperature optical conductivity is well described by the small bipolaron absorption, while the low temperature midinfrared band is due to absorption by small polarons. The bipolaron dissociation below $T_{c}$ shifts the spectral weight from the bipolaronic peak to the polaronic one. We describe the optical spectra of the layered ferromagnetic $\left(T_{c}=125 \mathrm{~K}\right)$ crystal $\mathrm{La}_{2-2 x} \mathrm{Sr}_{1+2 x} \mathrm{Mn}_{2} \mathrm{O}_{7}$ 18 in the entire frequency and temperature range that has been studied experimentally, and show that the optical data provides strong evidence for CCDC.

\section{FRÖHLICH INTERACTION AND SMALL (BI)POLARON FORMATION IN OXIDES}

The carriers in both cuprates and manganites are $\mathrm{O} p$ (not $\mathrm{Cu}$ or $\mathrm{Mn} d$ ) holes [22], and their pairing in the paramagnetic phase due to strong electron-phonon interaction would most likely be in the form of intersite bipolarons localized on a pair of O sites [1]. Clearly, the formation of these bipolarons cannot be hindered by repulsive on-site Hubbard- $U$ and/or Hund's rule coupling, 
contrary to recent claims in the literature [23].

In order to assess the possibility of small polaron and bipolaron formation in manganites and cuprates, one has to calculate the electron-phonon interaction and compare it with the (inter-site) Coulomb repulsion. Fortunately, such an estimate is possible in oxides, which are highly polarizable materials with a substantial longrange Fröhlich interaction. Then the polaron binding energy $E_{p}$ (polaronic shift) can be explicitly expressed through the well defined experimental parameters. In the long-wave limit the response of polarons at the optical phonon frequency is dynamic [24]. Their (renormalized) plasma frequency is lower than the optical phonon frequency due to the large static dielectric constant of oxides, an enhanced effective mass, and relatively low density of polarons. Therefore, the long-range character of the Fröhlich interaction is unaffected by screening. The optical phonon frequency is hardly affected as well [25. Claims in the literature [26,27] that the Fröhlich interaction is reduced to a local Holstein interaction in doped oxides disregard the established fact that the mobility of carriers determines the screening, rather than their density [1].

We shall apply the exact expression for the polaronic shift $E_{p}$ [28 30, 1], which for the Fröhlich interaction reads

$$
E_{p}=\frac{1}{2 \kappa} \int_{B Z} \frac{d^{3} q}{(2 \pi)^{3}} \frac{4 \pi e^{2}}{q^{2}} .
$$

Here the dielectric constants, $\kappa^{-1}=\epsilon_{\infty}^{-1}-\epsilon_{0}^{-1}$, are known from experiment, and the size of the integration region (the Brillouin zone, BZ) is determined by the lattice constants $a, b, c$ (Table). The data in the Table represent the lower boundary for the polaron binding energies, Eq. (1), since the account for coupling with acoustic and/or JahnTeller modes can only increase the polaronic shift $E_{p}$. The correct value of $E_{p}$ appears to be about $1 \mathrm{eV}$ in most cases, far exceeding the ad hoc estimates of $\sim 0.15 \mathrm{eV}$ [31, obtained from a numerically incorrect expression for $E_{p}$ and wrong values of the dielectric constants. The large calculated value of the polaron shift $(\sim 1 \mathrm{eV})$ is perfectly compatible with the small polaron theory. According to [31] the bare half-bandwidth is about $W / 2 \simeq 0.8$ $\mathrm{eV}$ in manganites. Hence, even a simple variational criteria [31] of the small polaron formation $\left(E_{p}>W / 2\right)$ is satisfied. In the case of the Fröhlich interaction the small polaron theory based on the Lang-Firsov canonical transformation is numerically accurate even in the weak coupling (large polaron) regime irrespective of the value of $E_{p}$ [32]. It is much more important, irrespective of any theoretical arguments, that the existence of polarons in CMR materials is unambiguosly confirmed experimentally, including: very low mobility [14,18], incompatible with Boltzmann-type approaches, activated dc and ac transport in the paramagnetic phase 18,33] and the giant isotope effect in manganites [10,11].
TABLE I. Polaron shift $E_{p}$ due to Fröhlich interaction. Data from Handbook of Optical Constants of Solids, edited by E.D. Palik (Academic, New York, 1997) and Landolt-Börnstein, Group III. The value $\epsilon_{\infty}=5$ for $\mathrm{WO}_{3}$ is an estimate.

\begin{tabular}{lllll}
\hline \hline System & $\epsilon_{\infty}$ & $\epsilon_{0}$ & $a \times b \times c\left(\AA^{3}\right)$ & $\mathrm{E}_{p}(\mathrm{eV})$ \\
\hline $\mathrm{BaBiO}_{3}$ & $5.7^{\mathrm{a}}$ & $30.4^{\mathrm{b}}$ & $4.34^{2} \times 4.32$ & 0.579 \\
$\mathrm{BaTiO}_{3}$ & $5.1-5.3$ & 1499. & $3.992^{2} \times 4.032$ & 0.842 \\
$\mathrm{La}_{2} \mathrm{CuO}_{4}$ & 5.0 & 30 & $3.8^{2} \times 6^{\mathrm{c}}$ & 0.647 \\
$\mathrm{LaMnO}_{3}$ & $3.9^{\mathrm{d}}$ & $16^{\mathrm{de}}$ & $3.86^{3}$ & 0.884 \\
$\mathrm{La}_{2-2 x} \mathrm{Sr}_{1+2 x}$ & & & & \\
$-\mathrm{Mn}_{2} \mathrm{O}_{7}$ & $4.9^{\mathrm{d}}$ & $38^{\mathrm{d}}$ & $3.86^{2} \times 3.9^{\mathrm{f}}$ & 0.807 \\
$\mathrm{SrTiO}_{3}$ & 5.2 & 310 & $3.905^{3}$ & 0.852 \\
$\mathrm{WO}_{3}$ & 5 & $100-300$ & $7.31 \times 7.54 \times 7.7$ & 0.445 \\
$\mathrm{CdO}$ & 5.4 & 21.9 & $4.7^{3}$ & 0.522 \\
$\mathrm{EuS}$ & 5.0 & 11.1 & $5.968^{3}$ & 0.324 \\
$\mathrm{EuSe}$ & 5.0 & 9.4 & $6.1936^{3}$ & 0.266 \\
$\mathrm{MgO}$ & 2.964 & 9.816 & $4.2147^{3}$ & 0.982 \\
$\mathrm{NaCl}$ & 2.44 & 5.90 & $5.643^{3}$ & 0.749 \\
$\mathrm{NiO}$ & 5.4 & 12 & $4.18^{3}$ & 0.429 \\
$\mathrm{TiO}$ & $6-7.2$ & $89-173$ & $4.59^{2} \times 2.96$ & 0.643 \\
\hline \hline
\end{tabular}

${ }^{a}$ S. Uchida et al. J. Phys. Soc. Jap. 54, 4395 (1985).

${ }^{b}$ R.P.S.M. Lobo and F. Gervais, Phys. Rev. B 52, 13294 (1995).

${ }^{\mathrm{c}}$ Distance between $\mathrm{CuO}_{2}$ planes.

${ }^{\mathrm{d}} \mathrm{T}$. Ishikawa (private communication, 1999).

${ }^{\mathrm{e}}$ J.H. Jung and T.W. Noh (private communication, 1999) have estimated from their data $\epsilon_{\infty}=3.4$ and $\epsilon_{0}=21$ for $\mathrm{LaMnO}_{3}$. ${ }^{\mathrm{f}}$ Distance between $\mathrm{MnO}_{2}$ planes.

The effective polaron-polaron attraction, due to the overlap of the deformation fields, is about $2 E_{p}$ [1]. This appears to be more than sufficient to overcome the intersite Coulomb repulsion, $V_{c} \simeq 0.7-0.8 \mathrm{eV}$, as also confirmed by the first-principles lattice minimization technique [34]. Hence, we can conclude that the electronphonon interaction is comparable or even stronger than any relevant magnetic interaction (which is estimated as $\lesssim 0.2 \mathrm{eV}[31$ ), so that small (bi)polarons are indeed the most probable quasiparticles in oxides, in contrast to the erroneous claims 31].

\section{OPTICAL CONDUCTIVITY OF INTERSITE BIPOLARONS}

Now we show how the observed massive weight transfer in optical conductivity with temperature [18] can be naturally explained within the bipolaron theory. The optical intraband conductivity of a charge-transfer doped insulator with (bi)polaronic carriers is the sum of the polaron $\sigma_{p}(\nu)$ and bipolaron $\sigma_{b}(\nu)$ contributions at a given frequency $\nu$. Their frequency dependences are described in the literature 335,36, 1, 37 40]. In the leading saddle-point approximation both have almost a Gaussian shape given by 
(a)

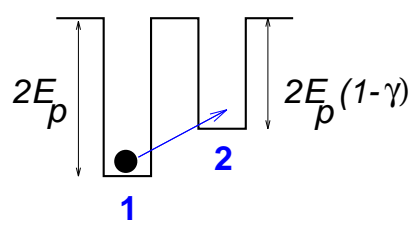

(b)

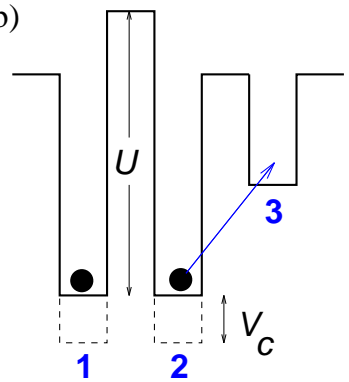

FIG. 1 Alexandrov and Bratkovsky

FIG. 1. Adiabatic energy levels and optical trannsitions for the small polaron (a) and the inter-site small bipolaron (b).

$$
\begin{aligned}
\sigma_{\text {intra }}(\nu) & =\frac{\sigma_{0} \mathcal{T}^{2}}{\nu}\left[\frac{n}{\gamma_{p}} \exp \left[-\left(\nu-\nu_{p}\right)^{2} / \gamma_{p}^{2}\right]\right. \\
& \left.+\frac{x-n}{\gamma_{b}} \exp \left[-\left(\nu-\nu_{b}\right)^{2} / \gamma_{b}^{2}\right]\right]
\end{aligned}
$$

where $\sigma_{0}=2 \pi^{1 / 2} e^{2} / a$ is a constant with $a$ the lattice spacing, $\mathcal{T}$ the hopping integral, $n$ the (atomic) polaron density, and $x$ the doping level. Here and further we take $\hbar=c=1$.

We shall first determine the positions and widths of the (bi)polaron absorption peaks for oxides. Those are known for the Holstein model with local interactions, but it is unlikely to apply for oxides because of very large on-site Coulomb repulsion and the long-range (Fröhlich) electron-phonon interaction, which dominates in ionic solids.

Applying the Franck-Condon principle 36] in the adiabatic regime, $\nu \gg \omega$ (phonon frequency), one can generalize the (bi)polaronic absorption, Eq. (2), to describe the optical conductivity of small Fröhlich (bi)polarons, Fig. 1. The electron 'sitting' on a site "1", Fig. 1(a), lowers its energy by an amount $2 E_{p}$, with respect to an atomic level in the undeformed lattice, owing to the lattice deformation. If the electron-phonon interaction has a finite radius, the electron also creates some deformation around a neighboring site " 2 ", lowering its energy level by an amount $2 E_{p}(1-\gamma)$, where 42]:

$$
\gamma=\sum_{\mathbf{q}}|\gamma(\mathbf{q})|^{2}[1-\cos (\mathbf{q} \cdot \mathbf{a})] / \sum_{\mathbf{q}}|\gamma(\mathbf{q})|^{2}
$$

with a the lattice vector connecting the neighboring sites. The coefficient $\gamma$ strongly depends on the radius of the interaction. In the Holstein model with $\mathbf{q}-$ independent electron-phonon coupling, $\gamma(\mathbf{q})$, this coefficient equals unity. Hence, there is no lattice deformation at the neighboring site. On the contrary, in the Fröhlich case, $\gamma(\mathbf{q}) \propto 1 / q$, and the coefficient is quite small, $\gamma \approx 0.2-0.442$ depending on the dimensionality of the system and the unit cell geometry. In that case, there is a significant lowering of the neighboring energy level and, as a result, of the polaron mass [32]. Hence, generally, the peak energy in the polaron absorption is found at

$$
\nu_{p}=2 \gamma E_{p}
$$

and the activation energy of the high-temperature dcconductivity is $E_{a}=\gamma E_{p} / 2$ 36]. One can apply the same 'frozen lattice distortion' arguments to the intersite bipolaron absorption, Fig. 1(b). The electron energy on site " 2 " is $-2 E_{p}-2 E_{p}(1-\gamma)+V_{c}$, where the first contribution is due to the lattice deformation created by the electron itself, while the second contribution is due to the lattice deformation around the site " 2 " created by the other electron of the pair on the site " 1 ", which is the polaron-polaron attraction [1]. After absorbing a quantum of radiation, the electron hops from site " 2 " to the empty site " 3 " into a state with the energy $-2 E_{p}(1-$ $\gamma$ ), which corresponds to an absorption frequency

$$
\nu_{b}=2 E_{p}-V_{c}
$$

where $V_{c}$ is the inter-site Coulomb repulsion. The quantum broadening of the polaronic and bipolaronic absorption is given by $\gamma_{p}=\gamma_{b}=\left(4 \gamma E_{p} \omega\right)^{1 / 2}$. Since doped manganites are intrinsically disordered, their dielectric properties are inhomogeneous, and so is $E_{p}$, which fluctuates with a characteristic impurity broadening $\Gamma_{\mathrm{im}}$. The convolution of the polaronic and bipolaronic absorption lines with the Gaussian distribution of $E_{p}$ results in their having different linewidths, $\gamma_{p}=2\left(\gamma E_{p} \omega+\gamma^{2} \Gamma_{\text {im }}^{2}\right)^{1 / 2}$ and $\gamma_{b}=2\left(\gamma E_{p} \omega+\Gamma_{\mathrm{im}}^{2}\right)^{1 / 2}$ for polaronic and bipolaronic absorption, respectively. The Coulomb repulsion $V_{c}$ can be readily estimated as $V_{c}=2 E_{p}-\nu_{b}$ from (5).

\section{SPECTRAL WEIGHT TRANSFER IN MANGANITES}

The total absorption is the sum of the intraband polaronic and bipolaronic terms, Eq. (2), and the interband absorption, $\sigma(\nu)=\sigma_{\text {intra }}(\nu)+\sigma_{\text {inter }}(\nu)$. In the layered compounds like $\mathrm{La}_{2-2 x} \mathrm{Sr}_{1+2 x} \mathrm{Mn}_{2} \mathrm{O}_{7}$, the intraband contribution to the out-of-plane conductivity is negligible 18]. Hence, one can take the c-axis optical conductivity $\sigma_{c}(\nu)$ as a measure of the interband contribution to the in-plane conductivity with a scaling factor, $s$, $\sigma_{\text {inter }}(\nu) \simeq s \sigma_{c}(\nu)$. The scaling factor $s$ is the square of 


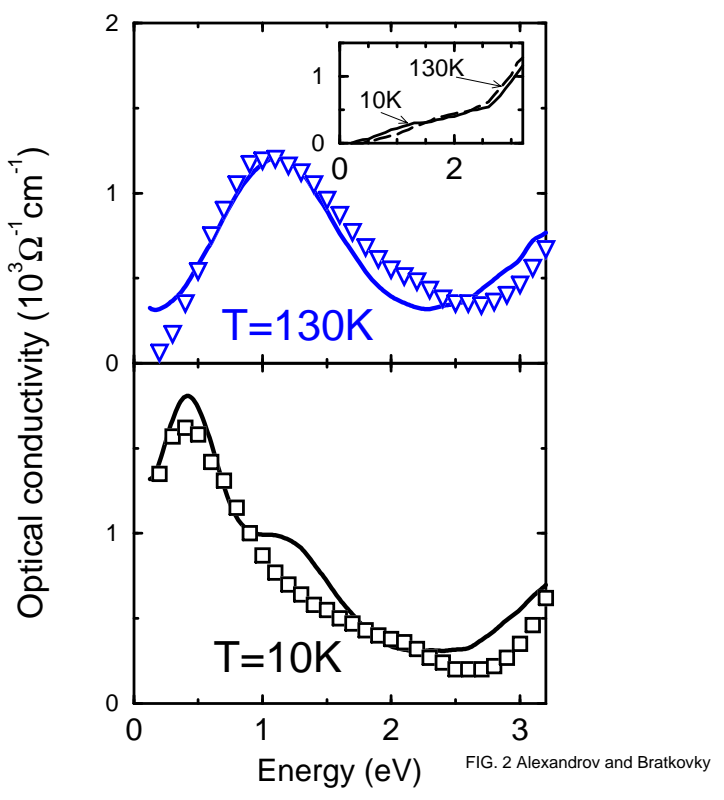

FIG. 2. Optical conductivity of $\mathrm{La}_{2-2 x} \mathrm{Sr}_{1+2 x} \mathrm{Mn}_{2} \mathrm{O}_{7}$ [17] compared with the theory (solid line) above $T_{c}$ (top panel) and well below $T_{c}$ (bottom panel). Inset: experimental c-axis optical conductivity [17].

the ratio of the in-plane components of the dipole matrix element for the interband transitions to its $z$ component ( $z$ is the out-of-plane direction). It can be readily determined by comparing the in-plane and out-of-plane optical conductivities at high frequencies, where intraband absorption is irrelevant. The result of the comparison of the present theory with the experiment [18] is shown in Fig. 2. At temperatures above the transition $(T=130 \mathrm{~K})$ the polaron density is very low owing to CCDC [5], so the intraband conductivity is due to bipolarons only,

$$
\sigma(\nu)=\frac{\sigma_{0} x \mathcal{T}^{2}}{\nu \gamma_{b}} \exp \left[-\left(\nu-\nu_{b}\right)^{2} / \gamma_{b}^{2}\right]+s \sigma_{c}(\nu)
$$

This expression fits the experiment fairly well with $\nu_{b}=1.24 \mathrm{eV}$ and $\gamma_{b}=0.6 \mathrm{eV}$, Fig. 2 . The scaling factor is estimated as $s=0.6$. When the temperature drops below $T_{c}$, at least some of the bipolarons break apart by the exchange interaction with Mn sites, because one of the spin-polarized polaron bands falls suddenly below the bipolaron level by an amount $\left(J_{p d} S-\Delta\right) / 2$, Fig. 3 [5]. The intraband optical conductivity is determined now by both the polaronic and bipolaronic contributions, Eq. (2), and that explains the sudden spectral weight transfer from $\nu=\nu_{b}$ to $\nu=\nu_{p}$, observed below $T_{c}$ in the ferromagnetic manganites [16 18]. The experimental spectral shape at $T=10 \mathrm{~K}$ is well described by Eq. (1) with $n=x / 5, \nu_{p} \simeq 0.5 \mathrm{eV}$ and $\gamma_{p} \simeq 0.3 \mathrm{eV}$ (Fig. 2). Taking $E_{p}=1 \mathrm{eV}$ (Table) we find from Eq.(4) $\gamma \simeq 0.25$ corresponding to the activation energy $E_{a}=125 \mathrm{meV}$ and phonon frequency $\omega \simeq 75-90 \mathrm{meV}$ [41]. With the use of Eq.(5) we find $V_{c} \simeq 0.76 \mathrm{eV}$ in good correspondence with previous estimates for cuprates [42].
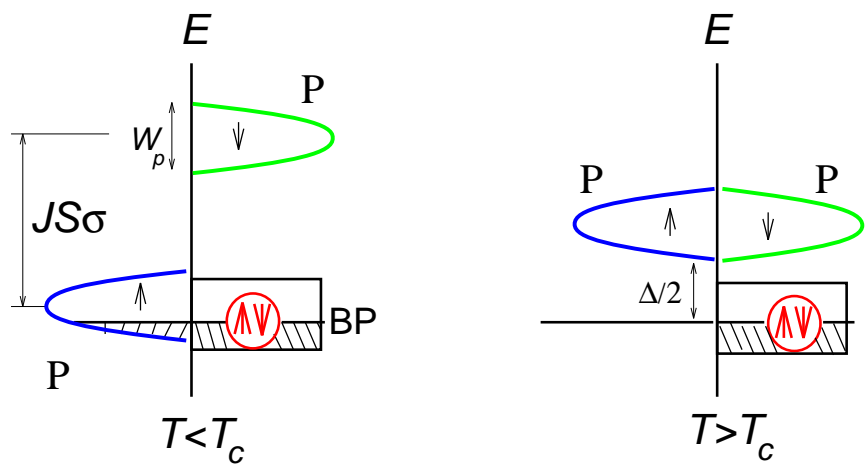

FIG. 3 Alexandrov and Bratkovsky

FIG. 3. Spin-polarized polaron band (P) in the ferromagnetic phase $\left(T<T_{c}\right)$ overlaps with the bipolaron (impurity) band (BP), breaking up a fraction of the bipolarons. In the paramagnetic phase $\left(T>T_{c}\right)$ the spin-splitting of the polaron band is absent and system contains mainly localized bipolarons.

We note the weak temperature dependence of the optical conductivity at low temperatures $T<50 \mathrm{~K}$ and above $T_{c}$ [18] in agreement with our theory. We do not expect any significant temperature dependence of the optical conductivity in the paramagnetic phase because the polaron density remains small compared with the bipolaron density above $T_{c}$ [5]. Our results also suggest a double-peak structure of $\sigma(\nu)$ below $T_{c}$, which was clearly observed for $\mathrm{La}_{0.7} \mathrm{Ca}_{0.3} \mathrm{MnO}_{3}$ [43]. This structure is not evident in the experiment on layered compound (Fig. 2), thus, more detailed studies of this low-temperature region are most desirable. It is worth mentioning that the scatter of the on-site energies of carriers may lead to an asymmetric broadening of both polaronic and bipolaronic absorption peaks [44], which can wash out the doublepeak structure at low temperatures.

The temperature dependence of the polaron density can be found from our Hartree-Fock equations [5 45]. The polaron density at zero temperature is about the total (chemical) density of holes introduced by doping, while in the paramagnetic phase one obtains

$$
n\left(T>T_{c}\right) \sim \exp \left(-\Delta / 2 k_{B} T\right)
$$

for $T \geq T_{c}$ with the prefactor depending on the ratio $W_{p} / T_{c}$. Note that $n \sim x$ below the Curie point, at $T<T_{c}$. Therefore, there is a very abrupt drop of the polaron density at $T_{c}$, which exponentially depends on the bipolaron binding energy $\Delta=\nu_{b}-\nu_{p}-W_{p}$ (Fig. 3). It can reach many orders of magnitude if the relative strength of the exchange interaction $J_{p d} S$ is close enough to the binding energy. 


\section{CONCLUSIONS}

In conclusion, we have calculated the value of the electron-phonon interaction in oxides (Table) to show that the Fröhlich interaction plays the dominant role in comparison with any magnetic (exchange) interaction. The conditions in oxides are such that small polarons and bipolarons are the most probable quasiparticles both in insulating and superconducting compounds. An opposite conclusion, recently suggested in the literature [31,26], stems from (i) an incorrect expression for the polaron binding energy, (ii) misunderstanding of the criteria for small polaron formation, and from (iii) misunderstanding of the screening in polaronic conductors. We have developed the theory of the optical conductivity in doped magnetic charge-transfer insulators with a strong electron-phonon interaction. The spectral and temperature features of the optical conductivity of ferromagnetic manganites are well described by the bipolaron absorption in the paramagnetic phase and by the small polaron absorption in the ferromagnetic phase. The pair breaking by exchange interaction with the localized Mn spins explains the sudden spectral weight transfer in the optical conductivity below $T_{c}$. Therefore, the optical probe of the incoherent charge dynamics in manganites provides another strong evidence for the carrier density collapse, which we proposed earlier as the explanation of CMR. The anomalous specific heat and tunneling measurements also support CCDC [9]. The Mn localised spins give a major contribution into magnetic susceptibility peak around $T_{c}$, and a deviation of its temperature dependence from standard Curie behavior may better elucidate a (smaller) magnetic response of polarons and a character of the phase transition.

Our picture is further supported by recent ARPES data [46], clearly showing a pseudogap in the band dispersion in manganites at the Fermi level at low temperatures, incompatible with the presence of a metallic phase. In addition, strong antiferromagnetic correlations in the paramagnetic phase at $T>T_{c}$, found in polarized neutron scattering and in Raman spectra [47] support our idea of the singlet pairing above $\mathrm{T}_{c}$. Outside the ferromagnetic regions (i) the bipolarons would tend to form charge density waves, usually observed as a charge ordering, and (ii) doped carries would spill over to $\mathrm{Mn}$ sites from oxygen [22], thus allowing, at least in principle, a possibility for some orbital effects. The theory suggests that by replacing the magnetic ions $(\mathrm{Mn})$ with nonmagnetic ions $(\mathrm{Cu})$, one can transform a doped charge-transfer insulator into a high-temperature superconductor owing to the Bose-Einstein condensation of bipolarons [1] , as was most likely observed experimentally 48$]$.

We are grateful to G. Aeppli, D.N. Basov, A.R. Bishop, D.S. Dessau, D.M. Eagles, T. Egami, J.P. Franck, F. Fu- jimori, P. Horsch, M.F. Hundley, T. Ishikawa, P.E. Kornilovitch, K.M. Krishnan, P.B. Littlewood, T.W. Noh, A.P. Ramirez, G. A. Sawatzky, R.S. Williams, J. Zaanen, R. Zeyher, and Guo-meng Zhao for helpful discussions. ASA acknowledges support from the Quantum Structures Research Initiative and the External Research Program of Hewlett-Packard Laboratories (Palo Alto).

* Email asa21@cus.cam.ac.uk

$\dagger \quad$ Email alexb@hpl.hp.com

[1] A.S. Alexandrov and N.F. Mott, Polarons and Bipolarons (World Scientific, Singapore, 1995).

[2] Anharmonic Properties of High- $T_{c}$ Cuprates, eds. D. Mihailović, G. Ruani, E. Kaldis, and K.A. Müller (World Scientific, Singapore,1995).

[3] A.J. Millis, P.B. Littlewood, and B.I. Shraiman, Phys. Rev. Lett. 74, 5144 (1995).

[4] H. Röder, Jun Zang, and A.R. Bishop, Phys. Rev. Lett. 76, 1356 (1996).

[5] A.S. Alexandrov and A.M. Bratkovsky, Phys. Rev. Lett. 82, 141 (1999).

[6] G.H. Jonker and J.H. Van Santen, Physica (Utrecht) 16, 337 (1950); J.H. Van Santen and G.H. Jonker, Physica (Utrecht) 16, 599 (1950).

[7] R. von Helmolt et al., Phys. Rev. Lett. 71, 2331 (1993).

[8] S. Jin et al., Science 264, 413 (1994).

[9] A.S. Alexandrov and A.M. Bratkovsky, J. Phys. Condens. Matter 11, 1989 (1999).

[10] Guo-meng Zhao et al., Nature 381, 676 (1996). It has been shown recently [Guo-meng Zhao et al., Phys. Rev. B 60, 11914 (1999)] that the giant isotope effect is intrinsic, while a reduced oxygen-isotope effect observed in the argon annealed samples may not be intrinsic.

[11] J.C. Irwin et al., Phys. Rev. B 59, 9362 (1999).

[12] A. Biswas et al., Phys. Rev. B 59, 5368 (1999).

[13] A.P. Ramirez, J. Phys. Condens. Matter 9, 8171 (1997).

[14] P. Schiffer et al., Phys. Rev. Lett. 75, 3336 (1995).

[15] Y. Okimoto et al., Phys. Rev. Lett. 75, 109 (1995).

[16] Y. Okimoto et al., Phys. Rev. B55, 4206 (1997).

[17] K.H. Kim, J.H. Yung, and T.W. Noh, Phys. Rev. Lett. 81, 1517 (1998).

[18] T. Ishikawa et al., Phys. Rev. B 57, R8079 (1998).

[19] D.S. Dessau et al., Phys. Rev. Lett. 81, 192 (1998).

[20] J.-S. Zhou et al., Phys. Rev. Lett. 79, 3234 (1997).

[21] In two dimensions $\sigma_{0}=\frac{e^{2}}{h}\left(k_{F} l\right)$.

[22] H.L. Ju et al., Phys. Rev. Lett. 79, 3230 (1997); G. A. Sawatzky (to be published).

[23] A.J. Millis, Nature (London) 392, 147 (1998).

[24] A.S. Alexandrov, Phys. Rev. B46, 2838 (1992).

[25] A.S. Alexandrov and J.R. Schrieffer, Phys. Rev. B 56, 13731 (1997).

[26] B.K. Chakraverty, J. Ranninger, and D. Feinberg, Phys. Rev. Lett. 81, 433 (1998).

[27] B.K. Chakraverty, J. Ranninger, and D. Feinberg, Phys. Rev. Lett. 82, 2621 (1999). 
[28] J. Yamashita and T. Kurosawa, J. Phys. Chem. Solids 5, 34 (1958).

[29] I.G. Lang and Yu.A. Firsov, Zh. Eksp. Teor. Fiz. 43, 1843 (1962) [Sov. Phys. JETP 16, 1301 (1963)].

[30] D.M. Eagles, Phys. Rev. 181, 1278 (1969).

[31] E.L. Nagaev, Phys. Lett. A258, 65 (1999).

[32] A.S. Alexandrov and P.E. Kornilovitch, Phys. Rev. Lett. 82, 807 (1999). In this work electrons were assumed to interact with ions, shifting in one direction, via usual dipole interaction. This is not an interaction with oscillating higher multipole moments, as erroneously claimed in Ref. 31.

[33] M. Jaime et al., Phys. Rev. Lett. 78, 951 (1997); J.P Franck et al., Phys. Rev. B 58, 5189 (1998); G. Jakob et al., Phys. Rev. B 58, 14966 (1998); L. Wang et al. Phys. Rev. B 60, R6976 (1999).

[34] C.R.A. Catlow, M.S. Islam, and X. Zhang, J. Phys.: Condens. Matter 10, L49 (1998).

[35] H. Böttger and V.V. Bryksin, Hopping Conduction in Solids (Akademie-Verlag, Berlin, 1985).

[36] G.D. Mahan, Many-Particle Physics (Plenum, New York, 1990).

[37] M.I. Klinger, Phys. Lett. 7, 102 (1963).

[38] D.M. Eagles, Phys. Rev. 130, 1381 (1963).

[39] H.G. Reik, Solid St. Commun. 1, 67 (1963).

[40] V.V. Bryksin and V.S. Voloshin, Fiz. Tverd. Tela (Leningrad) 26, 2357 (1984) [Sov. Phys. Solid State 26, 1429 (1984)].

[41] G. Aeppli (private communication, 1998); D.B. Romero, Bull. Stefan Univ. (La Jolla) 11, 399 (1999).

[42] A.S. Alexandrov, Phys. Rev. B 53, 2863 (1996).

[43] J.H. Jung et al., Phys. Rev. B 57, R11043 (1998).

[44] V.V. Bryksin, V.S. Voloshin, and A.V. Raitsev, Sov. Phys. Solid State 25, 820 (1983) [Fiz. Tverd. Tela 25, 1427 (1983)].

[45] A.S. Alexandrov and A.M. Bratkovsky, Phys. Rev. B 60, 6215 (1999).

[46] T. Saitoh et al. (to be published).

[47] G. Aeppli (unpublished), talk at the Ringberg workshop (Schloss Ringberg Tegernsee, Germany, 6-11 April, 1999); Y. Tokura (unpublished), ibid.

[48] J.G. Bednorz and K.A. Müller, Z. Phys. B64, 189 (1986). 